\title{
Spray-Dried Quercetin-Lactose Powders for Oral Tablets with Improved Dissolution Rates and Modified Material Properties
}

\author{
Long Li, ${ }^{1}$ Yuanwei Li, ${ }^{1}$ Zhiying Wu, ${ }^{1}$ Jinhui Chen, ${ }^{2}$ and Jia Chen $\mathbb{D}^{1}$ \\ ${ }^{1}$ Surgical Department of Urology, Hunan Provincial People's Hospital (The First-Affiliated Hospital of Hunan Normal University), \\ Changsha, Hunan 410005, China \\ ${ }^{2}$ Day Surgery Ward, Hunan Provincial People's Hospital (The First-Affiliated Hospital of Hunan Normal University), Changsha, \\ Hunan 410005, China
}

Correspondence should be addressed to Jia Chen; 202070201512@hunnu.edu.cn

Received 3 July 2021; Accepted 16 July 2021; Published 20 August 2021

Academic Editor: Songwen Tan

Copyright (C) 2021 Long Li et al. This is an open access article distributed under the Creative Commons Attribution License, which permits unrestricted use, distribution, and reproduction in any medium, provided the original work is properly cited.

\begin{abstract}
This study is aimed at using spray drying method to codisperse it with the commonly used drug carrier lactose in different solvents and then pass it through a spray dryer to obtain different samples. The results showed that the dissolution rate and solubility of the samples obtained by dispersion in hot water and $25 \%$ ethanol were significantly higher. The water of crystallization peaks of the raw material disappeared at $105-125^{\circ} \mathrm{C}$ and $130-150^{\circ} \mathrm{C}$ (DSC). The excipient lactose had a small upward exothermic peak at $177^{\circ} \mathrm{C}$ and a significant heat absorption peak at $209^{\circ} \mathrm{C}$ before untreated (XRD). $\alpha$-Lactose peaks were observed at $12.5^{\circ}, 19.1^{\circ}, 19.6^{\circ}$, and $19.9^{\circ}$ at $2 \theta$ in both samples, and $\beta$-lactose peaks were found at $10.5^{\circ}$ at $2 \theta$ in sample A, but not in sample B (FTIR). The complex sharp peaks of lactose at $1100 \mathrm{~cm}^{-1}$ and quercetin at $1700-1000 \mathrm{~cm}^{-1}$ became moderated (SEM). The sample obtained by spray drying has a slittype mesoporous structure with an average pore size of about $9.3 \mathrm{~nm}$.
\end{abstract}

\section{Introduction}

Quercetin (3,5,7-trihydroxy-2-(3,4-dihydroxyphenyl)-4Hchromen-4-one) is an important flavonoid; it is a bright citrus yellow needle-like crystal, completely insoluble in cold water but its solubility can be increased by heating, very soluble in solvents such as alcohol and lipids. It is one of the most common flavonoids in various foods and is found in a variety of fruits, vegetables, grains, and leaves, with green tea, apple, radish leaves, cranberries, apples, onions, buckwheat, and cilantro being among the more common types [1]. It is antioxidant, anti-inflammatory, antiatherosclerosis, antiobesity, antidiabetes, and other important beneficial effects on the human body [2-6]. However, because of its low solubility and absorption, its beneficial effects on the human body are very limited. In fact, preliminary pharmacokinetic studies of quercetin in humans have shown that the oral bioavailability of this compound is very low (complex metabolic reactions in the small intestine and stomach make it bioavailable, with a reported bioavailability of less than 10\%). Quercetin glucoside, the natural form of this compound, has an estimated absorption range of only $3 \%$ to $17 \%$ per $100 \mathrm{mg}$ of quercetin ingested in healthy individuals. The low bioavailability of quercetin is mainly due to its low absorption rate, extensive metabolism, and/or rapid elimination rate. After ingestion into the body, quercetin metabolites appear in plasma 30 minutes after ingestion but are excreted in large quantities within 24 hours [7]. This suggests that quercetin is rapidly cleared from the blood and has a short half-life. Numerous factors contribute to its practical application with significant difficulties and obstacles.

In recent years, several innovative methods for enhancing the bioavailability of insoluble quercetin have been developed [8-11]. Among them, quercetin-loaded solid lipid nanoparticles have been developed [12]. Compared with pure quercetin powder, their solubility has been improved, but they still have problems such as complicated preparation, high process requirements, and instability.

In the pharmaceutical industry, the aqueous solubility of drug candidates is a very important factor that hinders the entry of drugs into clinical applications. In general, the 
bioavailability of both BCS class II and class IV drugs is constrained by the degree of dissolution and dissolution rate. Therefore, a slight improvement in the rate of dissolution can sometimes lead to a significant increase in bioavailability. According to drug theory, dosage form is a very important factor in determining the dissolution properties of a drug. For drugs absorbed from the gastrointestinal tract, amorphous drug forms are effective in increasing the solubility of compounds, thus greatly increasing the bioavailability of these drugs [13]. Unlike crystalline APIs, amorphous APIs have a more disordered structure due to the lack of longrange ordering of molecules. This disorder increases the Gibbs free energy thereby reducing the need to overcome the lattice energy during the dissolution process [14]. It has been reported that an increase in saturated solubility of amorphous drugs may promote a remarkable increase in oral bioavailability. Spray drying technique [15-17] is an important method to obtain amorphous drugs. Spray drying is also a method to well mix multiple ingredients [18].

\section{Materials and Methods}

2.1. Materials. The quercetin was purchased from (purity $>96$ \%) Sarn Chemical Technology (Shanghai) Co. Ltd. The pharmaceutical grade $\alpha$-lactose monohydrate (purity $\geq 99.9 \%$ ) was purchased from Jiangsu Dawning Pharmaceutical Co., Ltd., China. The anhydrous ethanol (purity $\geq 99.7 \%$ ) was purchased from Shanghai Titan Technology Co. The purified water used in this experiment was made by the laboratory.

2.2. Experimental Design. In order to increase the solubility and dissolution rate of the insoluble quercetin, we used a spray drying technique in an attempt to convert quercetin and lactose crystals into an amorphous form and keep them stable over a period of time. Lactose is a normal excipient for drug loading [19]. Since quercetin is almost insoluble in cold water, its solubility can be increased by heating, very soluble in solvents such as alcohol and lipids, and in order to dissolve the API (quercetin) as much as possible to destroy its lattice structure, we dispersed the same proportion of quercetin and lactose mixture in $60^{\circ} \mathrm{C}$ hot water and $25 \%$ hot ethanol, respectively, and stirred them at $1000 \mathrm{rpm} / \mathrm{min}$ to dissolve and fully disperse them, respectively.

In this experiment, $200 \mathrm{ml}$ of purified water and $200 \mathrm{ml}$ of $25 \%$ ethanol were precisely measured and placed in a beaker and heated to $60^{\circ} \mathrm{C}$. Subsequently, $0.5 \mathrm{~g}$ of quercetin and $9.5 \mathrm{~g}$ of lactose were accurately weighed and dissolved in two beakers to obtain suspension $A$ and suspension B, respectively.

Two suspensions were dried using the identical spray drying parameters, and the spray dryer (Shanghai YC-015) was adjusted to the optimum parameters as follows: fan frequency $50.0 \mathrm{~Hz}$, inlet air temperature $140^{\circ} \mathrm{C}$, outlet air temperature $120^{\circ} \mathrm{C}$, peristaltic pump speed $12.0 \mathrm{ml} / \mathrm{min}$, spray pressure $0.20 \mathrm{MPa}$, and nozzle diameter $0.5 \mathrm{~mm}$. Spray dry the two suspensions separately to obtain sample A and sample B.

Tablets were prepared by manually introducing powders into the biconvex-faced punch of a hydraulic press with a compression pressure of $4 \mathrm{MPa}$. A desktop tableting machine was used, and the internal diameter of the equipped tableting die was $10.0 \mathrm{~mm}$ (equal to the diameter of the tablets).

2.3. Characterization of Samples. Dissolution samples were obtained from RC1210G Dissolution Tester (Xinzhi, China). The extraction method was paddle method, the speed was $75 \mathrm{rpm} / \mathrm{min}$, the temperature was $37 \pm 0.2^{\circ} \mathrm{C}$, and the sampling height was $500 \mathrm{~mL}$. UV-Vis absorption spectra were obtained at $200 \sim 400 \mathrm{~nm}$ with a UV-2401 pc spectrophotometer at Shimadzu, Kyoto, Japan. The obtained sample powders were analyzed by DSC using a differential scanning calorimeter (HSC-4 DSC, Henven, China). Samples for DSC determination were prepared using a sealed aluminum pan following standard procedures. Approximately $8.0 \mathrm{mg}$ of each specimen was used in the analysis. The specimen was heated from $25^{\circ} \mathrm{C}$ to $300^{\circ} \mathrm{C}$ at a rate of $5^{\circ} \mathrm{C} / \mathrm{min}$, using $\mathrm{N}_{2}$ as the purge gas. To analyze all samples, heat flow was recorded versus temperature rise [20].

The raw materials and samples (quercetin crystals, lactose, and physical mixture and samples A and B) were studied using Fourier transform infrared spectroscopy (FTIR). The samples were compounded with dried $\mathrm{KBr}$ powder, pressurized into transparent flakes, and scanned for transmission sensitivity in a Nicolet 6700 FTIR spectrometer (Thermo Fisher Scientific). The FTIR spectral resolution was $1 \mathrm{~cm}^{-1}$. The solid powder sample was placed on an aluminum sample peg on a carbon-band. The gold-plated samples were examined with a JSM-7200F scanning electron microscope (SEM, JEOL Ltd.). The samples were analyzed using a thermogravimetric analyzer (TGA Q5000 V3.17 Build 265). The samples were tested in an alumina pot with $\mathrm{N}_{2}$ as equilibrium gas [21]. The temperature was set at $35^{\circ} \mathrm{C} \sim 350^{\circ} \mathrm{C}$, and the heating rate was constant at $5^{\circ} \mathrm{C} / \mathrm{min}$, under pyrolytic conditions with nitrogen flow. The crystallization characteristics of the two sample specimen powders were studied by XRD analysis. The solid samples were mounted on powder cartridges and analyzed by a Siemens D5000 diffractometer with $1.0 \mathrm{~g}$ of sample dispersed in the cartridge. The scan area of diffraction angle $(2 \theta)$ was $5 \sim 50^{\circ}$, the scan rate was $1.2^{\circ} / \mathrm{min}$, the scan current was $30 \mathrm{~mA}$, and the scan voltage was $40 \mathrm{kV} . \mathrm{N}_{2}$ adsorption experiments were performed on the powder samples separately to evaluate their relative porosity.

2.4. Results and Discussion. We analyzed the data obtained from the experimental assay. The dissolution curves of the three powders for the same conditions are shown in Figure 1, and it can be seen that the untreated quercetin crystal tablets were almost insoluble under the present experimental conditions. The dissolution rate and dissolution degree of the two powders by spray drying increased significantly compared to quercetin crystals [22]. The solubilization effect of sample B obtained by $25 \%$ ethanol dispersion was more pronounced, and the dissolution rate was also faster. This may be due to the fact that more quercetin crystals were dissolved in suspension B, causing a large amount of quercetin crystal structure to be disrupted, and more amorphous quercetin was produced during the spraying operation, causing an increase in its solubility. This was also verified in the subsequent analysis. 


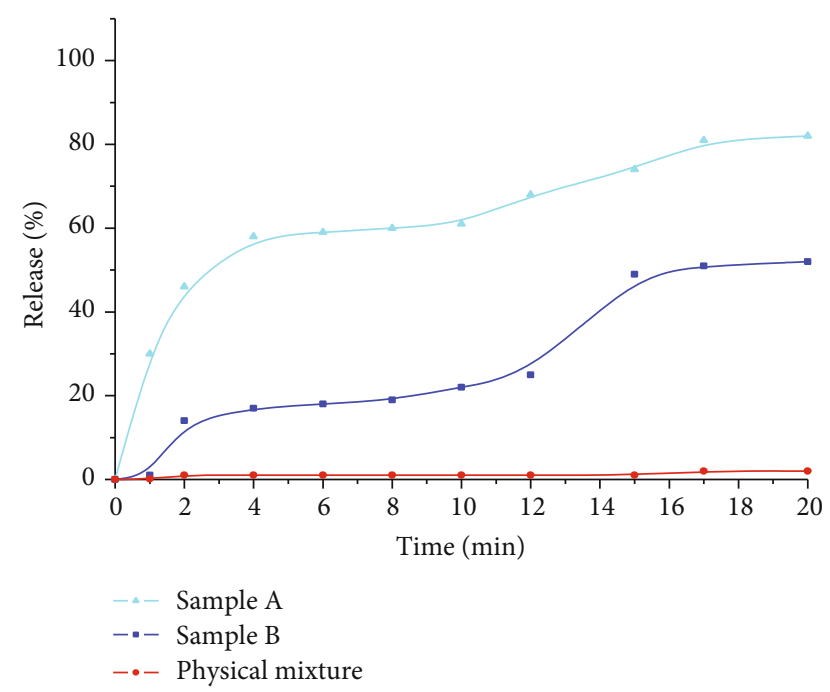

FIGURE 1: Release spectra of quercetin tablets in the two samples and physical mixture.

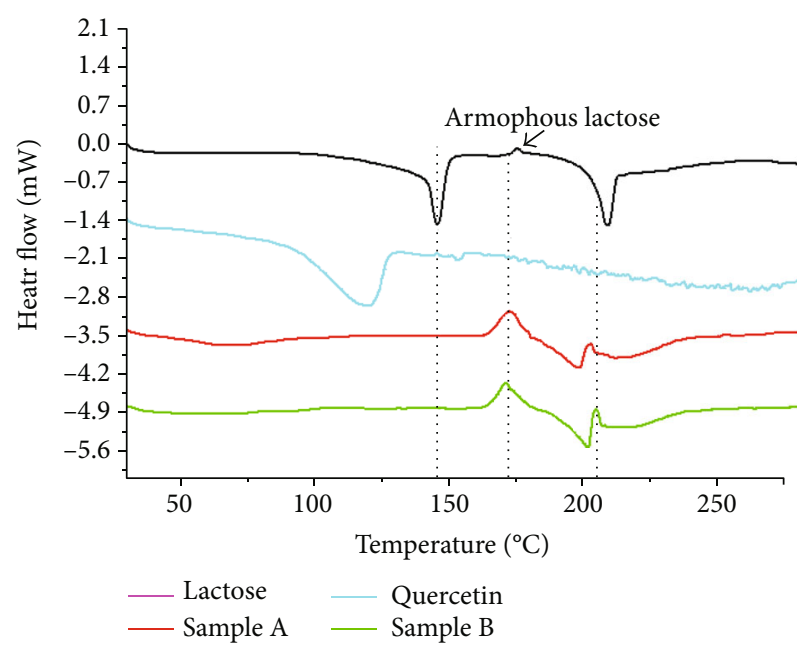

FiguRE 2: DSC spectrum of the raw materials and productions.

The DSC curve (Figure 2) shows that relative to the two spray-dried products. The water of crystallization peaks of the raw material disappeared at $105-125^{\circ} \mathrm{C}$ and $130-150^{\circ} \mathrm{C}$. The excipient lactose had a small upward exothermic peak at $177^{\circ} \mathrm{C}$ and a significant heat absorption peak at $209^{\circ} \mathrm{C}$ before untreated. However, after the spray drying operation with the API, the exothermic peak widened and moved to the low temperature region, indicating the formation of amorphous structure of lactose in the product. Meanwhile, the heatabsorbing peak of lactose at $209^{\circ} \mathrm{C}$ disappeared, but the heatabsorbing and exothermic peaks appeared simultaneously between 198 and $205^{\circ} \mathrm{C}$. By comparing the absorption curves of sample A and sample B, we found that the amorphous lactose exothermic peak of sample B shifted left relative to sample A, and the peak at $198-205^{\circ} \mathrm{C}$ shifted right relative to sample A. According to the analysis, it may be that more lactose but less API was dissolved in the pure water, resulting in more amorphous lactose generated and less amorphous quer-

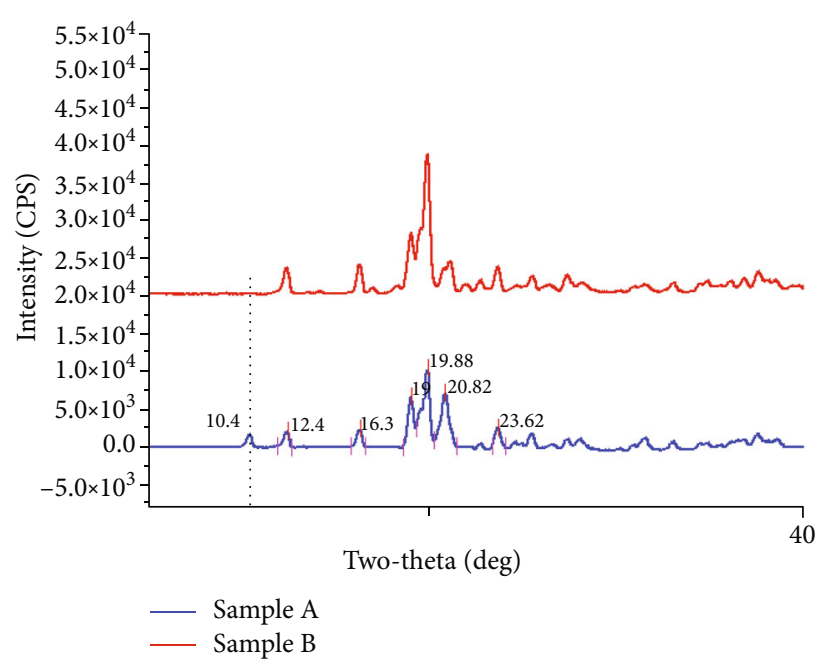

FIGURE 3: XRD spectrum of two samples.

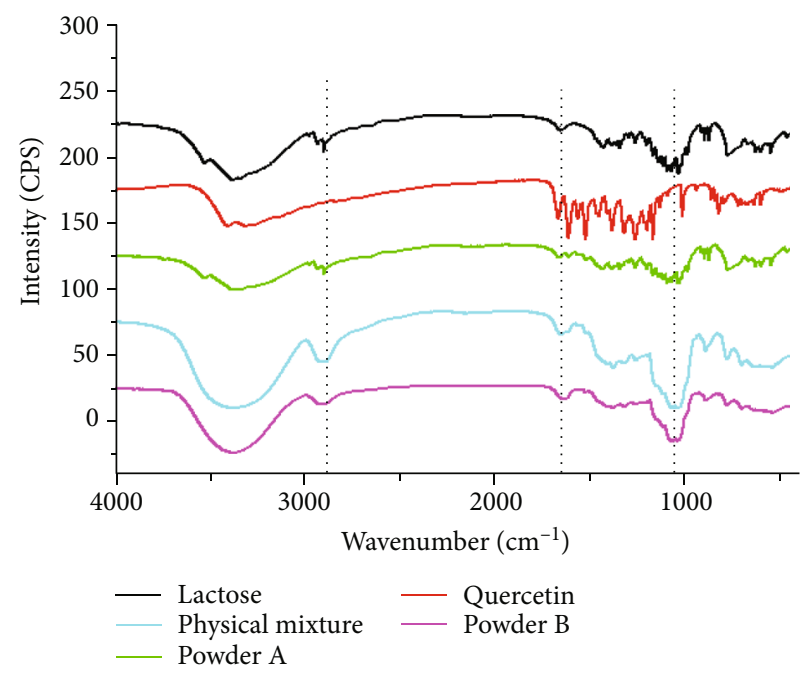

FIGURE 4: FTIR spectra of the raw materials and samples.

cetin generated. Interestingly, the disappearance of the small peak in the thermal spectrum of the quercetin API complex suggests that a homogeneous single mixture was obtained. According to the XRD spectra (Figure 3), $\alpha$-lactose peaks were observed at $12.5^{\circ}, 19.1^{\circ}, 19.6^{\circ}$, and $19.9^{\circ}$ at $2 \theta$ in both samples, and $\beta$-lactose peaks were found at $10.5^{\circ}$ at $2 \theta$ in sample $A$, but not in sample B. And the peak shape of both samples was flat, and the lactose peak of sample B was slightly sharper [16]. The analysis suggested that the higher content of amorphous product was obtained in sample A, which was consistent with the results of DSC analysis. The change in peak intensity or wave number shift was studied by FTIR (Figure 4). Similarly, the complex sharp peaks of lactose at $1100 \mathrm{~cm}^{-1}$ and quercetin at $1700-1000 \mathrm{~cm}^{-1}$ became moderated. This indicates a decrease in the density and strength of hydrogen bonds, a decrease in crystalline states, and a low level of intramolecular interactions. This leads to more dispersion of vibrational energy levels and lower conformational selectivity $[23,24]$. 

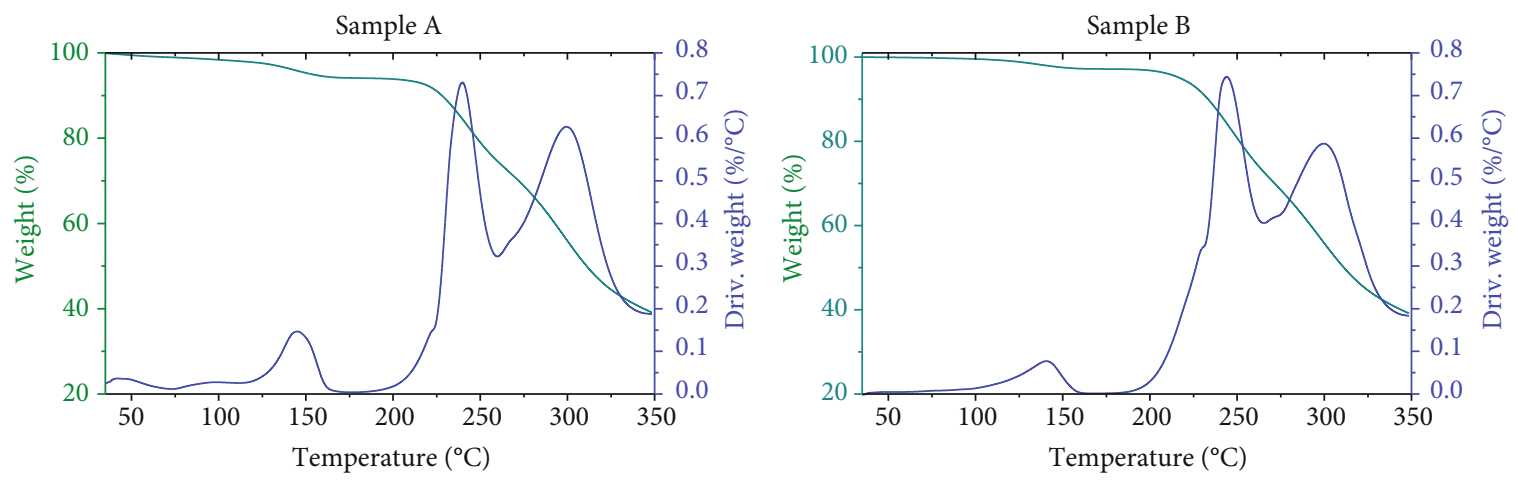

FIgURe 5: The TGA curve of the two samples.
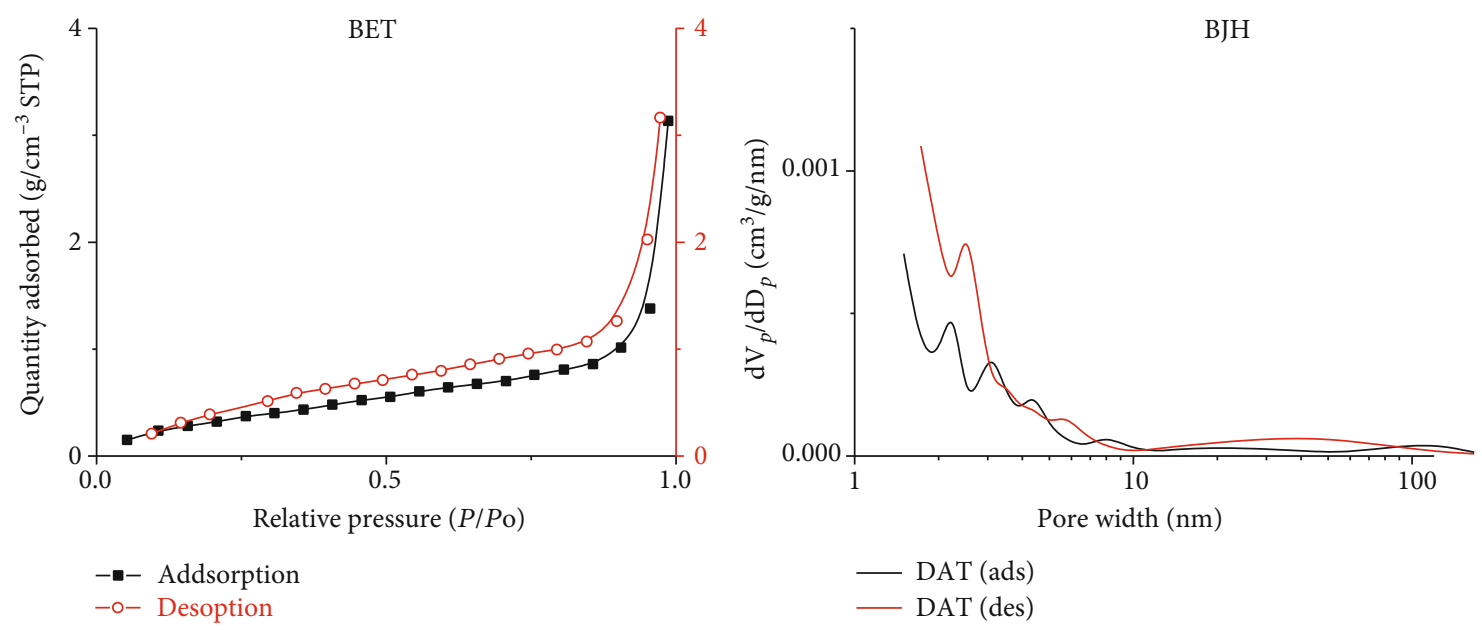

Figure 6: The $\mathrm{N}_{2}$ adsorption and desorption curves of the sample A.

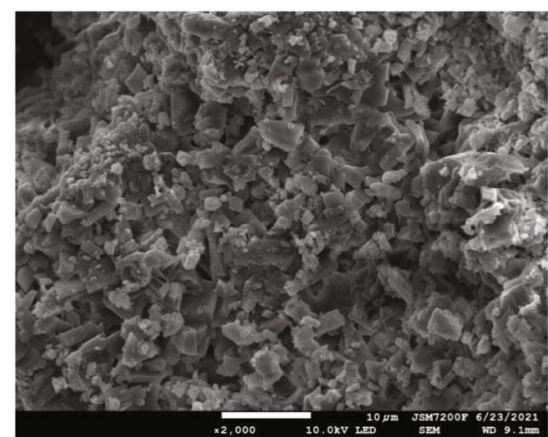

(a)

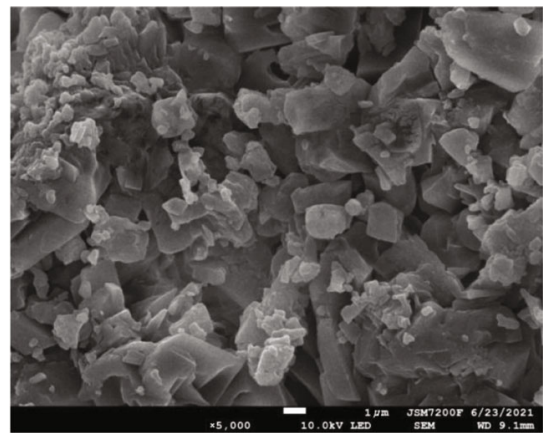

(c)

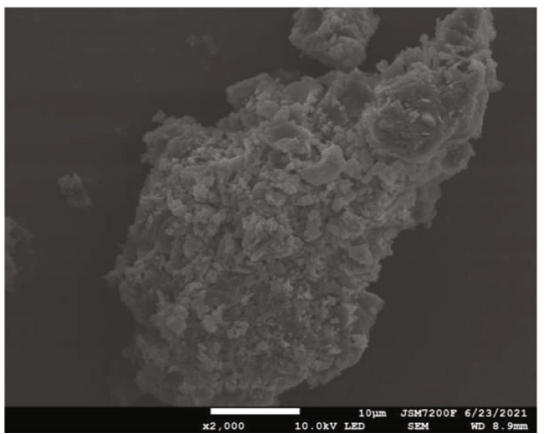

(b)

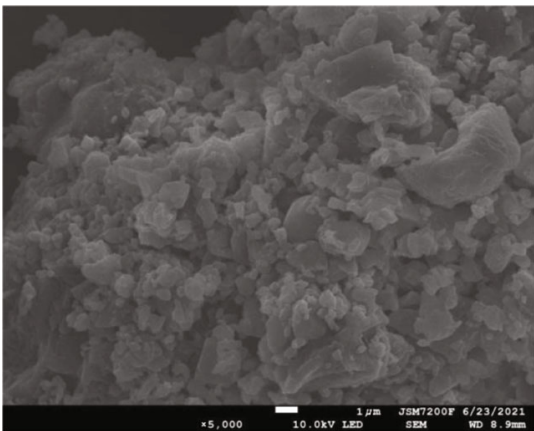

(d)

Figure 7: SEM images of the (a,b) sample A and (c, d) sample B, respectively. 
According to the TGA (Figure 5) curves, the weight loss rates of the two samples were basically the same in the same temperature range and the thermal stability was the same. Except for the sample containing a small amount of water, DTG analysis samples were roughly two-step weight loss, sample A had a slightly higher weight loss rate in the first step, and the analysis might be that it contained more amorphous lactose. According to the analysis of BET and $\mathrm{BJH}$ (Figure 6) data, the adsorption isotherm has a hysteresis loop, and isotherm of $\mathrm{H} 3$ type and the adsorption and desorption branches overlap at the cavitation point, and the preliminary analysis of the sample material has slit-type pores or macropores. Combined with the SEM (Figure 7) analysis, the sample obtained by spray drying has a slit-type mesoporous structure with an average pore size of about $9.3 \mathrm{~nm}$. Its porous structure can effectively promote the penetration of water, providing a guarantee for the rapid disintegration and dissolution of drugs.

\section{Conclusions}

In our experiments, we used pure water and $25 \%$ ethanol as the dispersion medium, followed by spray drying, respectively. The in vitro release characteristics were determined by pressing the tablets after atomization and drying. The solubility and dissolution rate of the samples obtained in both dispersion media were higher than those of the control group. In the two media, lactose and quercetin produced different dissolution characteristics such that after spray drying, both materials yielded different proportions of amorphous products. It was demonstrated that the solubility of quercetin increased after performing spray drying, and the solubilization effect was more pronounced for the sample obtained with $25 \%$ ethanol. The results showed that the dissolution rate and solubility of the samples obtained by dispersion in hot water and $25 \%$ ethanol were significantly higher. The water of crystallization peaks of the raw material disappeared at $105-125^{\circ} \mathrm{C}$ and $130-150^{\circ} \mathrm{C}$. The excipient lactose had a small upward exothermic peak at $177^{\circ} \mathrm{C}$ and a significant heat absorption peak at $209^{\circ} \mathrm{C}$ before untreated. $\alpha$-Lactose peaks were observed at $12.5^{\circ}, 19.1^{\circ}, 19.6^{\circ}$, and $19.9^{\circ}$ at $2 \theta$ in both samples, and $\beta$-lactose peaks were found at $10.5^{\circ}$ at $2 \theta$ in sample A, but not in sample B. The complex sharp peaks of lactose at $1100 \mathrm{~cm}^{-1}$ and quercetin at $1700-1000 \mathrm{~cm}^{-1}$ became moderated. Scanning electron microscopy images and BET data showed that the two samples obtained by spray drying contained incompletely dissolved crystals, which played a positive role in the formation of mesoporous structures, which further increased the dissolution rate. In conclusion, the spray drying technique significantly improved the solubility of quercetin and provided an idea to solve the problem of its low bioavailability.

\section{Data Availability}

The data used to support the findings of this study are included within the article.

\section{Conflicts of Interest}

The authors declare that they have no conflicts of interest.

\section{Acknowledgments}

This work was supported by the Hunan Natural Science Foundation (Grant Nos. 2020JJ4401 and 2017JJ3180) and the Scientific Research Project of Hunan Provincial Department of Education (Grant No. 20C1158).

\section{References}

[1] U. Shabbir, M. Rubab, E. B.-M. Daliri, R. Chelliah, A. Javed, and D.-H. Oh, "Curcumin, quercetin, catechins and metabolic diseases: the role of gut microbiota," Nutrients, vol. 13, no. 1, p. 206, 2021.

[2] H. Khan, H. Ullah, M. Aschner, W. S. Cheang, and E. K. Akkol, "Neuroprotective effects of quercetin in Alzheimer's disease," Biomolecules, vol. 10, no. 1, p. 59, 2020.

[3] Y. Li, J. Yao, C. Han et al., "Quercetin, inflammation and immunity,” Nutrients, vol. 8, no. 3, p. 167, 2016.

[4] D. Xu, M.-J. Hu, Y.-Q. Wang, and Y.-L. Cui, “Antioxidant activities of quercetin and its complexes for medicinal application," Molecules (Basel, Switzerland), vol. 24, no. 6, p. 1123, 2019.

[5] P. Fernández-Palanca, F. Fondevila, C. Méndez-Blanco, M. J. Tuñón, J. González-Gallego, and J. L. Mauriz, “Antitumor effects of quercetin in hepatocarcinoma in vitro and in vivo models: a systematic review," Nutrients, vol. 11, no. 12, p. 2875, 2019.

[6] X. Liu, Y. Zhang, L. Liu et al., "Protective and therapeutic effects of nanoliposomal quercetin on acute liver injury in rats," BMC Pharmacology and Toxicology, vol. 21, no. 1, p. 11, 2020.

[7] W. M. Dabeek and M. V. Marra, "Dietary quercetin and kaempferol: bioavailability and potential cardiovascularrelated bioactivity in humans," Nutrients, vol. 11, no. 10, p. 2288, 2019.

[8] J. S. Nam, A. R. Sharma, L. T. Nguyen, C. Chakraborty, G. Sharma, and S. S. Lee, "Application of bioactive quercetin in oncotherapy: from nutrition to nanomedicine," Molecules, vol. 21, no. 1, article E108, 2016.

[9] Y. Zhou, G. Quan, Q. Wu et al., "Mesoporous silica nanoparticles for drug and gene delivery," Acta Pharmaceutica Sinica $B$, vol. 8, no. 2, pp. 165-177, 2018.

[10] K. T. J. Chen, M. Anantha, A. W. Y. Leung et al., "Characterization of a liposomal copper (II)-quercetin formulation suitable for parenteral use," Drug Delivery and Translational Research, vol. 10, no. 1, pp. 202-215, 2020.

[11] H. Y. Son, M. S. Lee, E. Chang et al., "Formulation and characterization of quercetin-loaded oil in water nanoemulsion and evaluation of hypocholesterolemic activity in rats," Nutrients, vol. 11, no. 2, p. 244, 2019.

[12] O. Lushchak, O. Strilbytska, A. Koliada et al., "Nanodelivery of phytobioactive compounds for treating aging-associated disorders," GeroScience, vol. 42, no. 1, pp. 117-139, 2020.

[13] A. Schittny, J. Huwyler, and M. Puchkov, "Mechanisms of increased bioavailability through amorphous solid dispersions: a review," Drug Delivery, vol. 27, no. 1, pp. 110-127, 2020. 
[14] B. Wang, F. Liu, J. Xiang et al., "A critical review of spray-dried amorphous pharmaceuticals: synthesis, analysis and application," International Journal of Pharmaceutics, vol. 594, article ???, 2021.

[15] S. Tan, A. Ebrahimi, X. Liu, and T. Langrish, "Role of templating agents in the spray drying and postcrystallization of lactose for the production of highly porous powders," Drying Technology, vol. 36, no. 15, pp. 1882-1891, 2018.

[16] S. Tan, T. Jiang, A. Ebrahimi, and T. Langrish, "Effect of spraydrying temperature on the formation of flower-like lactose for griseofulvin loading," European Journal of Pharmaceutical Sciences, vol. 111, pp. 534-539, 2018.

[17] S. Tan, C. Zhong, and T. Langrish, "Encapsulation of caffeine in spray-dried micro-eggs for controlled release: the effect of spray-drying (cooking) temperature," Food Hydrocolloids, vol. 108, article ???, 2020.

[18] S. Tan, X. Chen, S. Zhai, A. Ebrahimi, T. Langrish, and Y. Chen, "Spray drying assisted synthesis of porous carbons from whey powders for capacitive energy storage," Energy, vol. 147, pp. 308-316, 2018.

[19] S. Tan, A. Ebrahimi, X. Liu, and T. Langrish, "Hollow flowerlike lactose particles as potential drug carriers: effect of particle size and feed concentration," Powder Technology, vol. 320, pp. 1-6, 2017.

[20] B. Wang, H. Li, J. Xiang, J. Zheng, and J. Wang, "Fabrication of agglomerated lactose using fluidized bed for good compressibility," Journal of Nanomaterials, vol. 2021, Article ID 9918847, 6 pages, 2021.

[21] J. Zheng, B. Wang, J. Xiang, and Z. Yu, "Controlled Release of Curcumin from HPMC (Hydroxypropyl Methyl Cellulose) Co- Spray-Dried Materials," Bioinorganic Chemistry and Applications, vol. 2021, Article ID 7625585, 6 pages, 2021.

[22] M. Buchweitz, P. A. Kroon, G. T. Rich, and P. J. Wilde, "Quercetin solubilisation in bile salts: a comparison with sodium dodecyl sulphate," Food Chemistry, vol. 211, pp. 356-364, 2016.

[23] Z. Hajihashemi, A. Nasirpour, J. Scher, and S. Desobry, "Interactions among lactose, $\beta$-lactoglobulin and starch in colyophilized mixtures as determined by Fourier Transform Infrared Spectroscopy," Journal of Food Science and Technology, vol. 51, no. 11, pp. 3376-3382, 2014.

[24] M. A. Ottenhof, W. Mac Naughtan, and I. A. Farhat, "FTIR study of state and phase transitions of low moisture sucrose and lactose," Carbohydrate Research, vol. 338, no. 21, pp. 2195-2202, 2003. 